\title{
Thermomechanical Simulation of an Aerogel/RTV Based Cryogenic Propellant Tank
}

\author{
Dalton Bowen ${ }^{1}$ \\ University of Memphis, Memphis, Tennessee 38111, USA
}

\begin{abstract}
Developing an effective solution for long duration storage of cryogenic liquids is critical for future, manned space exploration missions. Current storage tanks are made of metals such as steel, aluminum or composites. Although these materials have high mechanical strength, their relatively high thermal conductivity is a disadvantage with regards to heat infiltration into the cryogenic liquid. The influx of heat into the tank causes the cryogenic liquid to vaporize, increasing the pressure in the tank. To regulate the tank pressure, current tanks vent some of the vapor. To reduce tank pressurization rates, novel materials with lower thermal conductivities such as RTV-655 and aerogels have been developed which may be feasible for space applications. Previous experiments with small-scale RTV-655 and Aerogel/RTV-655 tanks were performed to obtain stress and strain histories as a function of temperature and pressure. Due to the complexity and costs of performing additional experiments, a thermomechanical computational model is desired to further study the feasibility of using these novel materials for space applications. A thermochemical finite element simulation is used to model the cooldown and pressurization phases of the RTV-655 and RTV-655/Aerogel experiments. Simulation predictions are presented and compared to the experiment measurements.
\end{abstract}

\section{Nomenclature}

$\begin{array}{lll}\text { RTV } & = & \text { Room Temperature Vulcanized } \\ \text { FEA } & = & \text { Finite Element Analysis } \\ \alpha\left(\theta, \mathrm{f}_{\mathrm{B}}\right) & = & \text { thermal expansion coefficient } \\ \theta & = & \text { current temperature } \\ \theta^{\mathrm{I}} & = & \text { initial temperature } \\ \mathrm{f}_{\beta} & = & \text { current values of field variables } \\ \mathrm{f}_{\mathrm{I}} & = & \text { initial values of field variables } \\ \theta^{0} & = & \text { reference temperature } \\ \epsilon_{\mathrm{m}} & = & \text { simulated strain } \\ \epsilon_{\mathrm{e}} & = & \text { experimental strain } \\ \mathrm{n} & = & \text { number of time steps } \\ \sigma_{\mathrm{c}} & = & \text { circumferential stress }\end{array}$

\section{Introduction}

For the next generation of manned spacecraft to be successful, the ability to store cryogenics needs to be greatly enhanced. Currently, cryogenic propellant tanks use metals or composites with relatively high thermal conductivities which leads to higher boiloff rates. Metal tanks also are susceptible to a process called "microcracking". Microcracking refers to the process of microscale crack formation in a material over time, weakening it. This microcracking can occur in metal and composite propellant tanks due to repeated self-pressurization and venting cycles associated with boiloff which causes the tanks to expand and contract repeatedly. Once these microcracks form, the tank can be compromised

\footnotetext{
${ }^{1}$ Undergraduate Student, Department of Mechanical Engineering, AIAA Student Member
} 
and can fail. Metals also have a relatively high density compared other materials, adding to the weight of the spacecraft, requiring more cryogenics for fuel[1].

To help overcome the disadvantages associated with metals and composites, novel materials which have recently been developed are being considered for cryogenic liquid storage applications. RTV-655 is a room-temperature vulcanized (RTV) elastomer with a relatively low thermal conductivity and may be a possible candidate for future cryogenic propellant tanks. Another possible material is polyimide aerogel. Crosslinked aerogels have relatively low densities and relatively low thermal conductivities, and some variants, such as polyimide aerogel, can be made into thin, flexible layers. When encapsulated in a polymer, such as RTV-655, it has potential to be an additional insulator in the tank. Both these materials have lower densities as compared to metals which will allow for lighter spacecraft. Experiments were previously conducted on two small-scale prototype cryogenic tanks. One of the tanks was made of RTV-655 while the other was made of RTV-655 with two encapsulated layers of polyimide aerogel. The experiments provided preliminary evidence that the RTV-655 and polyimide aerogel can maintain mechanical integrity and relatively low thermal conductivity at cryogenic temperatures. The results of the experiment supported continued investigation of the potential of using these materials for cryogenic liquids applications, such as cryogenic propellant tanks. A model of these tanks needs to be made in order to help expedite the process of understanding if these tanks are good candidates for the next generation of cryogenic storage tanks[1].

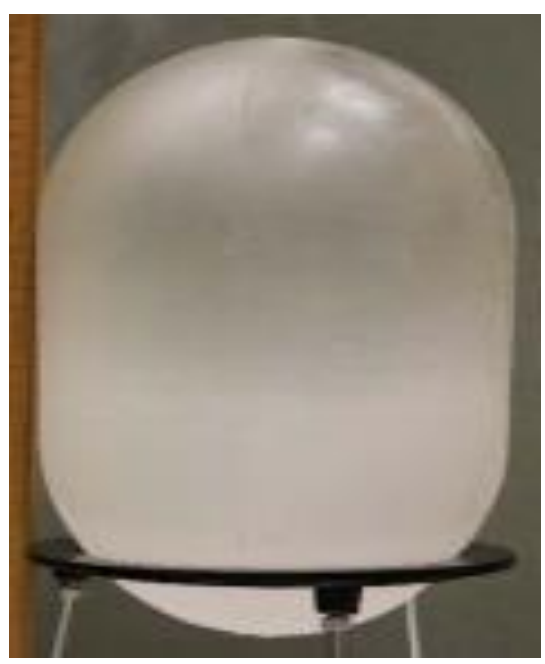

Figure 1: RTV Tank [2]

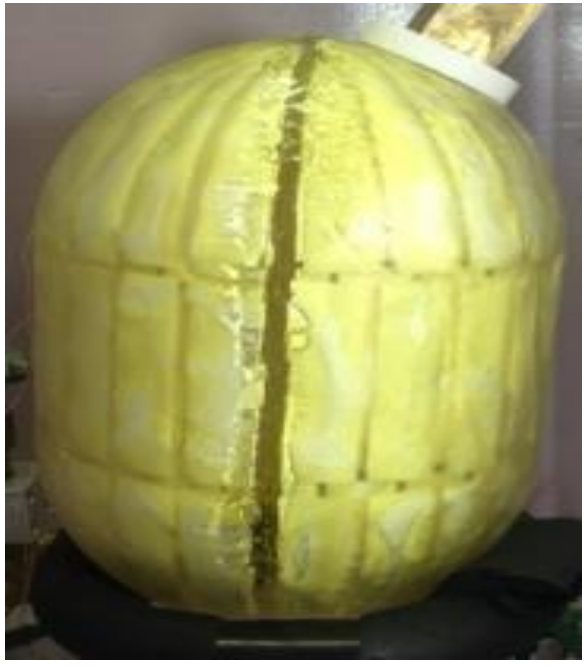

Figure 2: RTV/Aerogel Tank [2]

\section{Experimental Setup and Procedure}

Both prototype tanks were cylindrical pressure vessels with two hemispherical ends and were geometrically similar with the only main difference being that there is a $0.0018 \mathrm{~m}$ layer of aerogel in the RTV/Aerogel tank. The cylindrical section has a height of $0.102 \mathrm{~m}$ and the hemispherical ends have a radius of $0.102 \mathrm{~m}$. The tank's wall thickness is $0.019 \mathrm{~m}$ and has a ratio of 0.23 between its wall thickness and outside diameter which classifies it as a "thick walled" pressure vessel. After each tank is made, stems and fittings are added to allow for the flow of liquid nitrogen into the tank and allow the liquid nitrogen vapor to escape the tanks during boiloff. Type-K thermocouples are attached where the cylindrical portion of the tank meets the hemispherical ends on the inside and outside of each tank A diagram of the RTV/Aerogel tank can be seen in Figure 3[1]. 


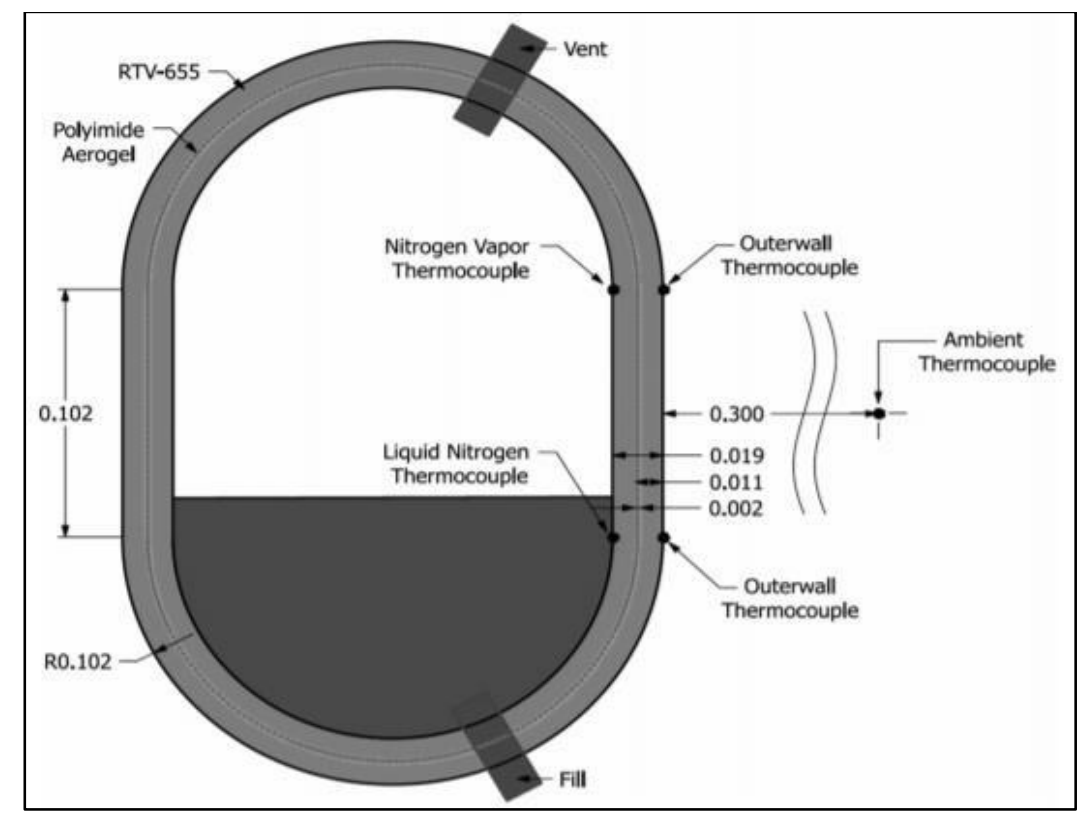

Figure 3: Cross-section of Tank [1]

For the experiment, each tank was placed in a testing frame and the piping for the liquid nitrogen is connected to the union fittings on the tank. The testing frame containing the tank was then placed into an insulated fume hood. The fume hood fan was turned off during the experiment but could be turned on to vent the chamber if necessary Three extensometers that measure the circumferential, diametral, and axial stains of the tank were attached. The liquid nitrogen supply is connected to the valve found at the bottom of the tank. The experimental setup of the RTV-655 and RTV/Aerogel tanks can be seen in Figure 4.

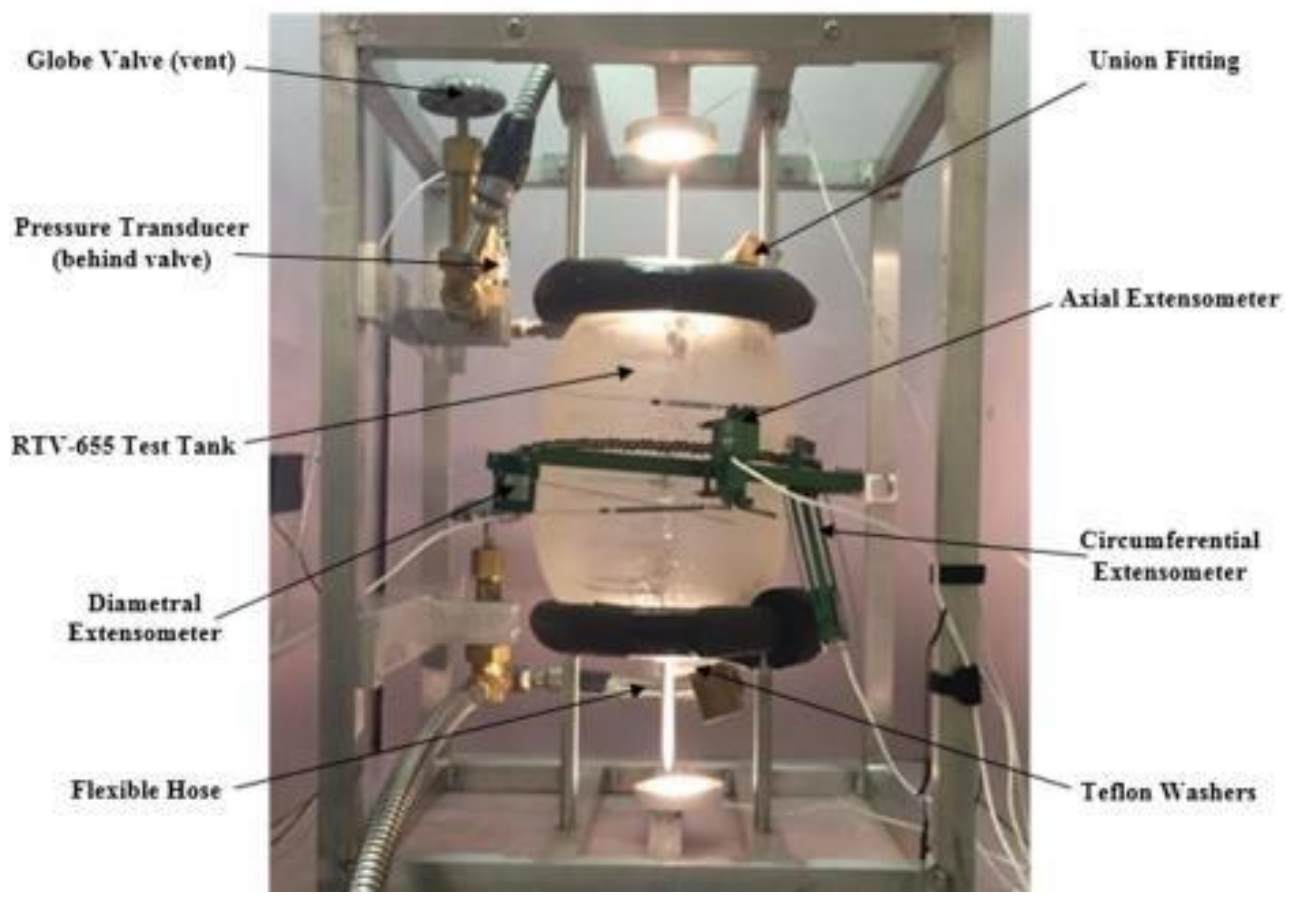

Figure 4: Experimental setup for RTV-655 Tank [1]

American Institute of Aeronautics and Astronautics 
Each test had four phases. The first phase, the Cooldown phase, consisted of slowly allowing liquid nitrogen to enter the tank. The liquid nitrogen would quickly vaporize and then escape out of the vent at the top of the tank into the fume hood chamber. The denser nitrogen vapor displaced the air in the chamber through an opening near the bottom of the fume hood chamber. Nitrogen that fell from the vent opening at the top of the tank to the bottom of the fume hood chamber cooled the exterior walls of the tank. Once the tank cooled to cryogenic temperatures, the liquid nitrogen began filling the tank. For these experiments, the tank was initially filled to $25 \%$ of the total tank volume. Once the $25 \%$ tank fill was achieved, the second phase, Pressurization, began. In this phase, the liquid nitrogen supply and vent are shut. The boiling of the liquid nitrogen allowed for pressure to build up in the tank. The maximum pressure the tank reached was 17 psig before a pressure relief safety valve opened. Once the safety valve opened, the third phase of keeping a Constant Liquid Fill began. This was achieved by reopening the supply and vents and adjusting the flow of liquid nitrogen into the tank to match the boiloff rate. Once this liquid level was maintained for approximately twenty minutes, the final phase, Boiloff, began. The supply of liquid nitrogen was slowed and eventually shutoff allowing the temperature of the tank to rise and the liquid nitrogen to be vaporized. The temperatures and strains were recorded during each phase. The simulation will attempt to model the Cooldown phase in order to perfect the boundary conditions before moving on to the more complicated stages of the experiment.

\section{FEA MODEL}

\section{A. RTV Tank Model/Mesh}

The RTV Tank was the first to be modeled because it was constructed of only one material. A commercial finite element analysis (FEA) software package[4] with the capability of modeling thermomechanical systems was used to simulate the experiment in the RTV-655 tank. The meshed tank, shown in Figure 5, has 28,664 elements and 35,840 nodes. A mesh convergence was conducted on the mesh to ensure that the solution was mesh independent. The first step was to simulate the Cooldown phase of the RTV-655 tank and validate it using the experimental strain data. Temperatures of the inner and outer walls recorded during the experiment, were used as the boundary conditions for the model. The tank is constrained in the model by applying a zero-rotation condition to the entire tank and a zero displacement to the bottom of the tank. The material properties used for the preliminary model of the RTV-655 tank are presented in Table 1 and 2.

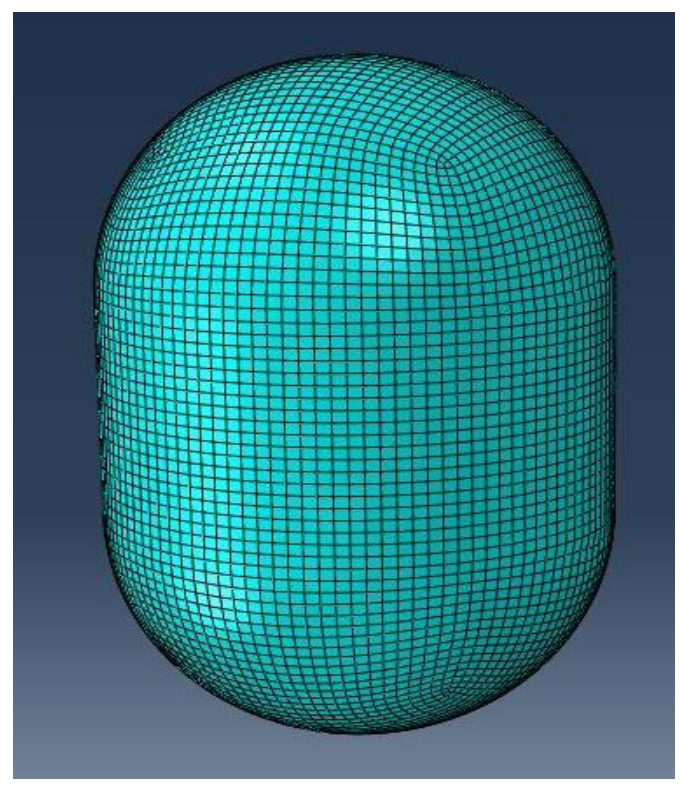

Figure 5: Meshed RTV Tank 
Table 1. Temperature Independent Properties for Preliminary Model

\begin{tabular}{|c|c|}
\hline Mass Density $\left(\mathbf{k g} / \mathbf{m}^{\mathbf{3}}\right)$ & 1040 \\
\hline Expansion Coefficient, $\boldsymbol{\alpha}(\mathbf{m} / \mathbf{m} / \mathbf{K})$ & $3.30 \mathrm{E}-04$ \\
\hline Specific Heat $(\mathbf{J} /(\mathbf{k g} * \mathbf{K}))$ & 1460 \\
\hline
\end{tabular}

Table 2. Temperature Dependent Properties for Preliminary Model

\begin{tabular}{|c|c|c|c|}
\hline $\begin{array}{c}\text { Young's Modulus } \\
(\mathbf{P a})\end{array}$ & Poisson's Ratio & $\begin{array}{c}\text { Thermal } \\
\text { Conductivity } \\
\left(\mathbf{W} /\left(\mathbf{m}^{*} \mathbf{K}\right)\right)\end{array}$ & Temp (K) \\
\hline $2.08 \mathrm{E}+08$ & 0.495 & .0833 & 298 \\
\hline $9.65 \mathrm{E}+05$ & 0.495 & .1843 & 75 \\
\hline
\end{tabular}

Thermal strains are calculated using the formula below.[4]

\section{B. Cooldown Phase Predictions}

$$
\epsilon^{\text {th }}=\alpha\left(\theta, f_{\mathrm{B}}\right)\left(\theta-\theta^{0}\right)-\alpha\left(\theta^{I}, f_{\mathrm{B}} I\right)\left(\theta^{I}-\theta^{0}\right)
$$

\section{B1. Constant Expansion Coefficient}

RTV-655 is a polymer which has well characterized material properties at room temperature, $298 \mathrm{~K}$. Property data for RTV-655 at temperatures below 298K, however, is limited. For the Cooldown phase, it is expected that most of the strains will be thermal strains and highly dependent on the coefficient of thermal expansion. A preliminary simulation was conducted using the constant expansion coefficient in Table 1. The simulation results can be seen in Figures 6-8. Since the expansion coefficient is constant, the strain is increases linearly with the temperature. This behavior does not match the experimental results.

\section{B2. Temperature Dependent Expansion Coefficients}

For the Cooldown phase, most of the strains will be thermal strains, so the thermal expansion coefficient must be accurate across the temperature range of interest. Instead of conducting time intensive and potentially costly experiments to determine the expansion coefficient directly over a range of temperatures, a literature review was conducted to find cryogenic tests on polymeric materials which exhibit thermochemical behavior similar to that of RTV-655. The U.S Department of Commerce released a compilation of literature on the "Thermal Expansion of Technical Solids at Low Temperatures" [3]. In this paper, the strain for a wide variety of materials was recorded as function of temperature. One of the materials tested was a "rubber silastic" which exhibits elastomeric behavior similar to RTV-655. Since the expansion coefficient is the slope of the strain versus temperature graph, the behavior of the expansion coefficient as a function of temperature was found and normalized for RTV-655 using the expansion coefficient of RTV-655 at 298K. The normalized expansion coefficient values are presented in Table 3.

Table 3: Table of Temperature Dependent Expansion Coefficients

\begin{tabular}{|c|c|}
\hline Temp (K) & $\begin{array}{c}\text { Derived Expansion } \\
\text { Coefficient }(\mathbf{m} / \mathbf{m} / \mathbf{K})\end{array}$ \\
\hline 280 & 0.00033 \\
\hline 240 & 0.000355 \\
\hline 200 & 0.000317 \\
\hline 160 & 0.000241 \\
\hline 120 & 0.000171 \\
\hline 80 & 0.000121 \\
\hline 40 & $5.71 \mathrm{E}-05$ \\
\hline
\end{tabular}


After the material properties were updated with the temperature dependent expansion coefficients, a simulation was performed, and the three component strain results are shown in Figures 12-15. The results can be seen in Figures 12-15. The temperature dependent expansion coefficients helped improve the model predictions in the axial and diametral directions. However, the simulation still underpredicts the circumferential strain by an order of magnitude below $-50^{\circ} \mathrm{C}$. This difference is not overly concerning since there were reports of problems with the circumferential strain extensometers during the experiment. Unlike the diametral and axial extensometers which utilized lubricated, thin guide wires and surface contact blades, the circumferential extensometer utilized a bulky chain and link to measure displacement. During the experiments, the metal chain stiffened considerably as frost formed on the outside surface of the tank and on the chain and the chain was observed to slip down from its vertical position on the outer surface of the tank when the tank contracted during the Cooldown phase.

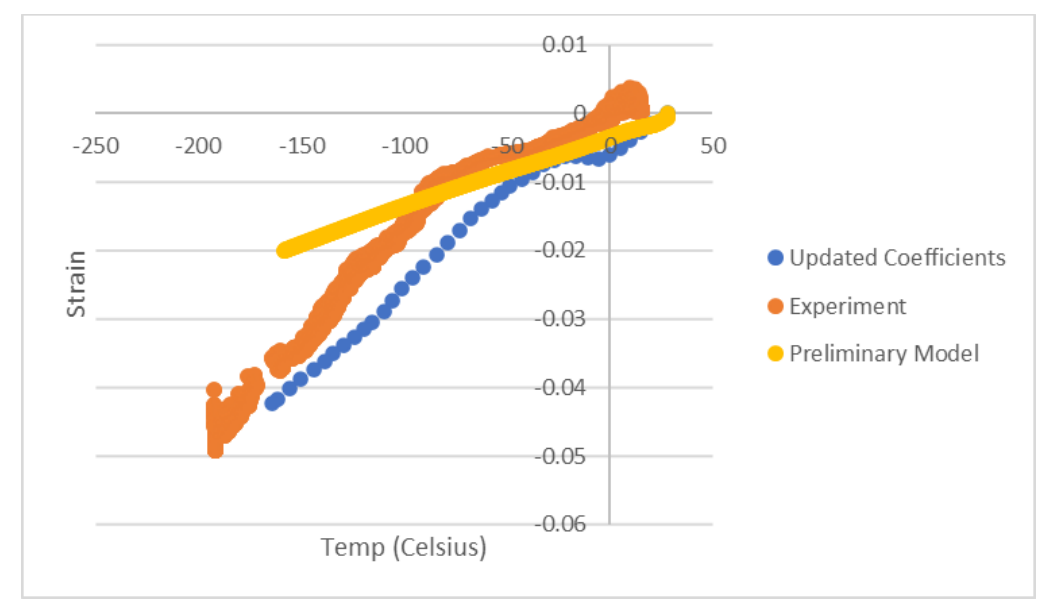

Figure 6: Comparison of Cooldown strains for the updated model and the experiment in the axial direction

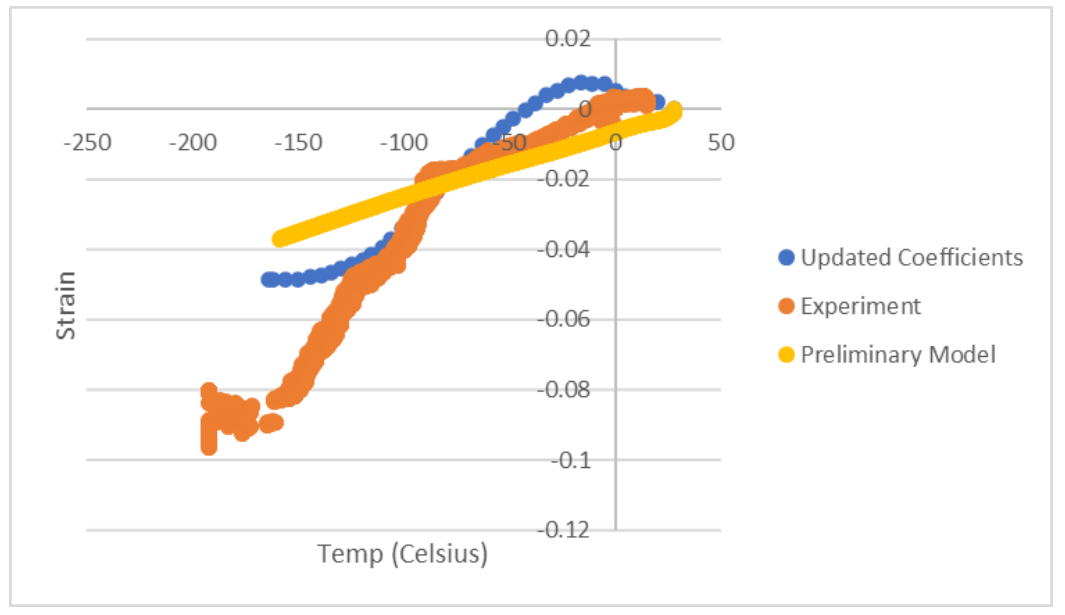

Figure 7: Comparison of Cooldown strains for the updated model and the experiment in the diametral direction 


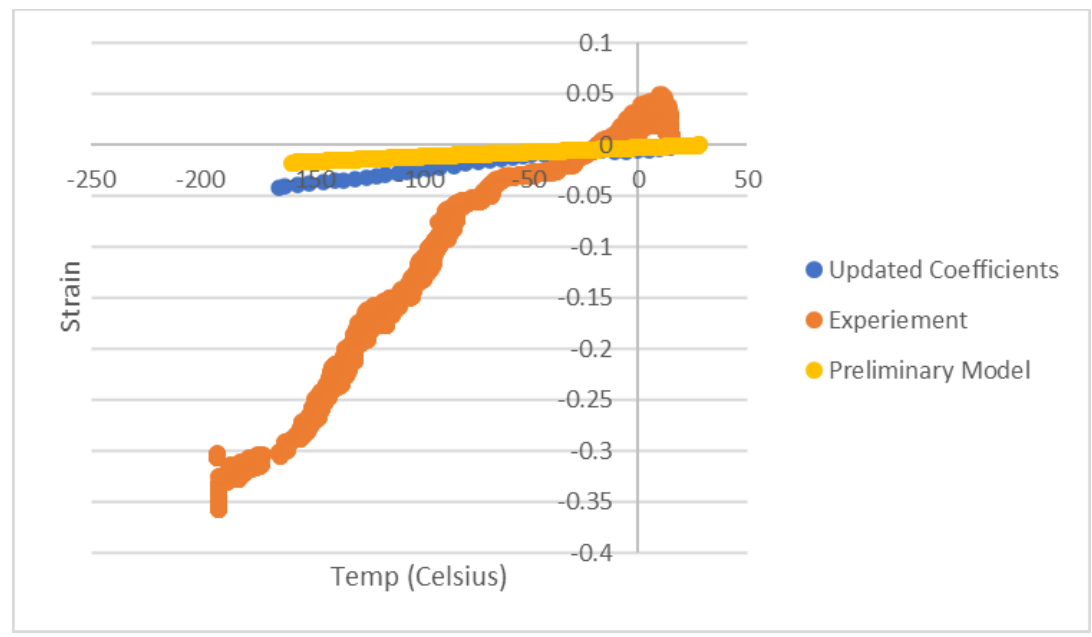

Figure 8: Comparison of Cooldown strains for the updated model and the experiment in the circumferential direction

\section{Pressurization Phase}

\section{D1. Two-point Young's Modulus}

After the completion of the Cooldown phase, the Pressurization phase was simulated. The same material properties from the Cooldown phase were used including the updated thermal expansion coefficient. The temperature was held constant for this phase, and the tank pressure was recorded during the experiment. The force due to the internal tank pressure was applied uniformly on the inner tank wall. The mechanical strains in the model were determined by using the two Young's Modulus points given at specific temperatures. The model would then linearly interpolate between the two points in order to calculate the mechanical strain caused by the pressurization of the tank. The results can be seen below in Figure 9.

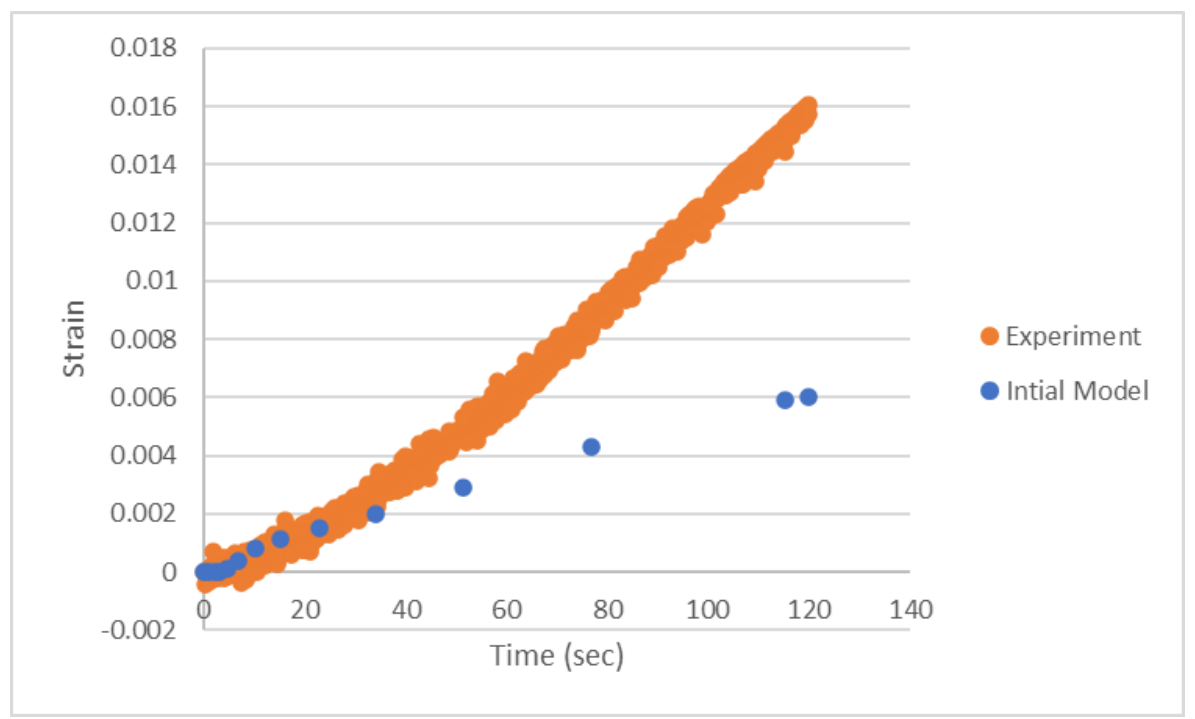

Figure 9: Total Strain Comparison of between Experimental Results and Model

American Institute of Aeronautics and Astronautics 


\section{D2. Hyperelasticity}

The inaccuracy of the predictions in the previous section were expected since most of the strain in this phase is no longer driven by the thermal strains, but rather the mechanical strains. It was reasonable to consider that RTV-655 is more hyperelastic than elastic. A hyperelastic solver was subsequently used to model the relationship between stress and strain. There are many different types of hyperelastic solvers, but the Marlow solver was chosen because it was the only solver available that will allow stress and strain data as a function of temperature. Stress and strain data at two different temperatures, $77 \mathrm{~K}$ and $298 \mathrm{~K}$, for RTV-655 was provided to the solver and a simulation was performed again. However, the results obtained using the hyperelastic model were underestimate the strain by two orders of magnitude. It was subsequently concluded that RTV-655 is not a hyperelastic material.

\section{D3. Two-point Young's Modulus with Modified Glass Transition Point}

Once it was determined that RTV-655 did not exhibit significant hyperelastic behavior, the focus returned to the two-point elastic model. A key feature of mechanical elastomer behavior which was not considered in the previous twopoint model was the glass transition temperature. The glass transition temperature is the relatively small temperature region at which the elastomer gets significantly stiffer or flexible. This temperature region can be very different from elastomer to elastomer and identifying it was crucial for improving the accuracy of this model. The specification sheet from the supplier of RTV-655 states that RTV-655 is elastic until it reaches a temperature of $158 \mathrm{~K}$. This temperature was then assumed to be the glass transition temperature region, and the Young's Modulus behavior was normalized using the two known values at 77K and 298K. A graph of the normalized RTV-655, and other elastomers can be seen below in Figure 10. A simulation was run using this behavior and the results can be seen in Figure 11. The results from this simulation were compared to the results of the two-point Young's Modulus using the normalized sum of square value for each simulation. The normalized sum of square values were $1.4 * 10^{-5}$ and $3.58^{*} 10^{-6}$ for the two-point Young's Modulus and the modified glass transition point simulations respectively. This means the glass transition temperature improved the accuracy of the model. [5]

Normalized sum of squares calculated using formula below:

$$
\frac{\sum_{i=0}^{n}\left(\epsilon_{m}-\epsilon_{\mathrm{e}}\right)^{2}}{n}
$$

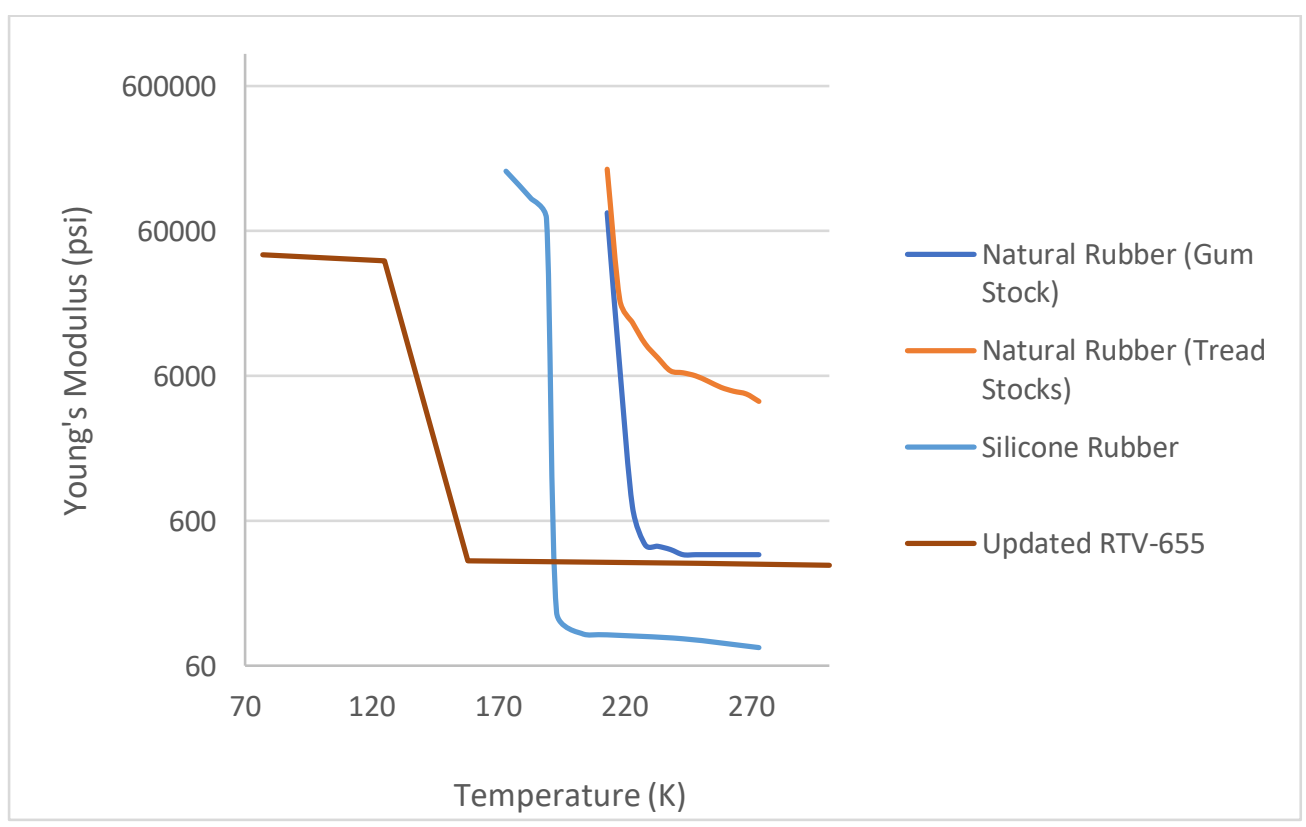

Figure 10: Comparison of Young's Modulus for different elastomers 


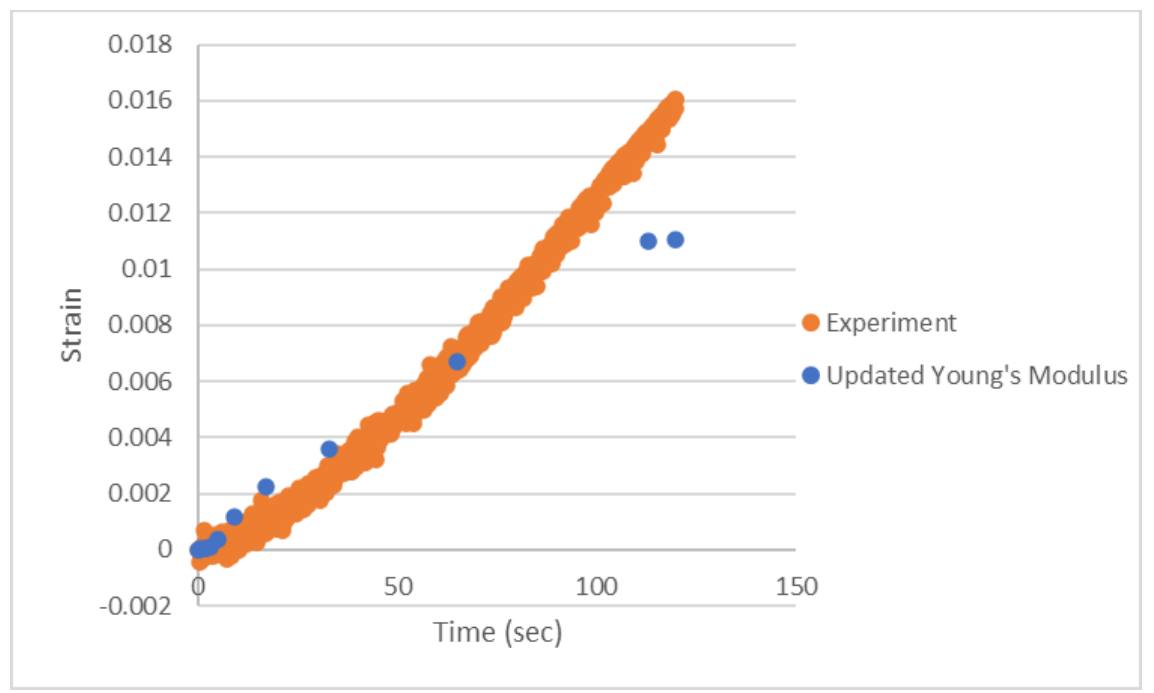

Figure 11: Total Strain Comparison between Experimental Results and Model with updated Young's Modulus

\section{Poisson's Ratio}

Another important material property for calculating mechanical strains is Poisson's Ratio. The previous models assumed the Poisson's Ratio was constant. A literature review was done on the temperature dependence of Poisson's Ratio for elastic materials. Several studies show that Poisson's Ratio is a function of temperature for some elastic and viscoelastic materials and changes rapidly around the glass transition temperature. Since the glass transition temperature for RTV-655 is known, the Poisson's ratio was updated to a function of temperature with a behavior similar to those found in the study and a simulation was conducted. The results can be seen below in Figure 12. The normilized sum of the squares values were used again to compare the accuracy of the this model to the previous model. The normilized sum of the square value of the temperature dependent Poisson's Ratio was $1.72 * 10^{-6}$ which is a smaller value than the previous model meaning the changes improved the accuracy of the model.[6]

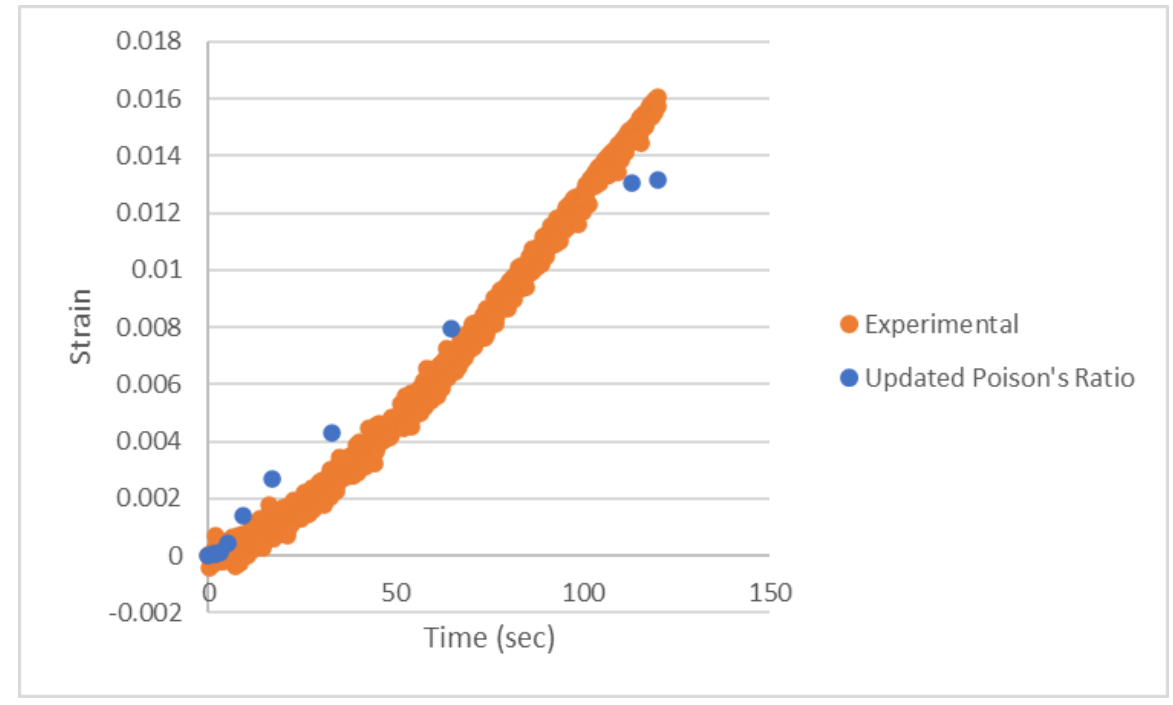

Figure 12: Total Strain Comparison Between Experiment and Model with updated Poisson's Ratio

American Institute of Aeronautics and Astronautics 


\section{E. Effect of Improved Mechanical Properties on the Cooldown Phase}

After the significant changes to the material properties that dominate the mechanical strain, it is important to revisit the Cooldown phase in order to see their effects. Although the thermal stains dominate the Cooldown phase, there is still some mechanical strain produced from contraction due to the geometry of the tank. These mechanical strains will now have higher values due to the updated Young's Modulus. The effect of the mechanical strains on the Cooldown phase can be seen in Figures 13-15. The results show that the Young's Modulus has a bigger effect than initially realized. These material property changes greatly improve the accuracy in the diametral strain results, but overestimate the axial strain.

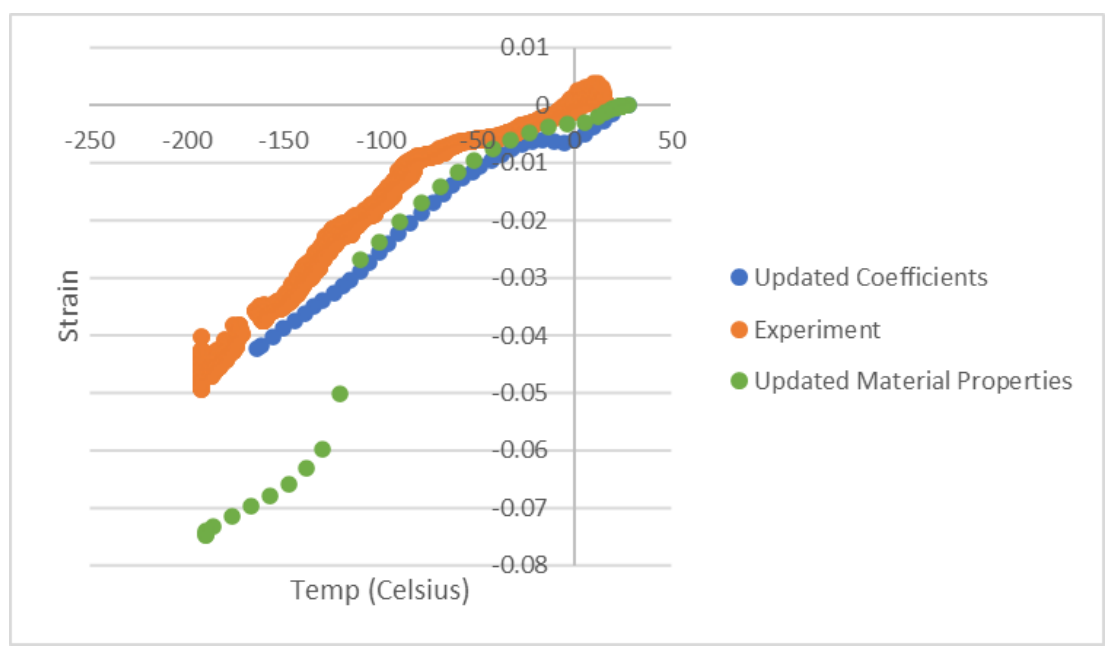

Figure 13: Comparison of cooldown strains in the axial direction

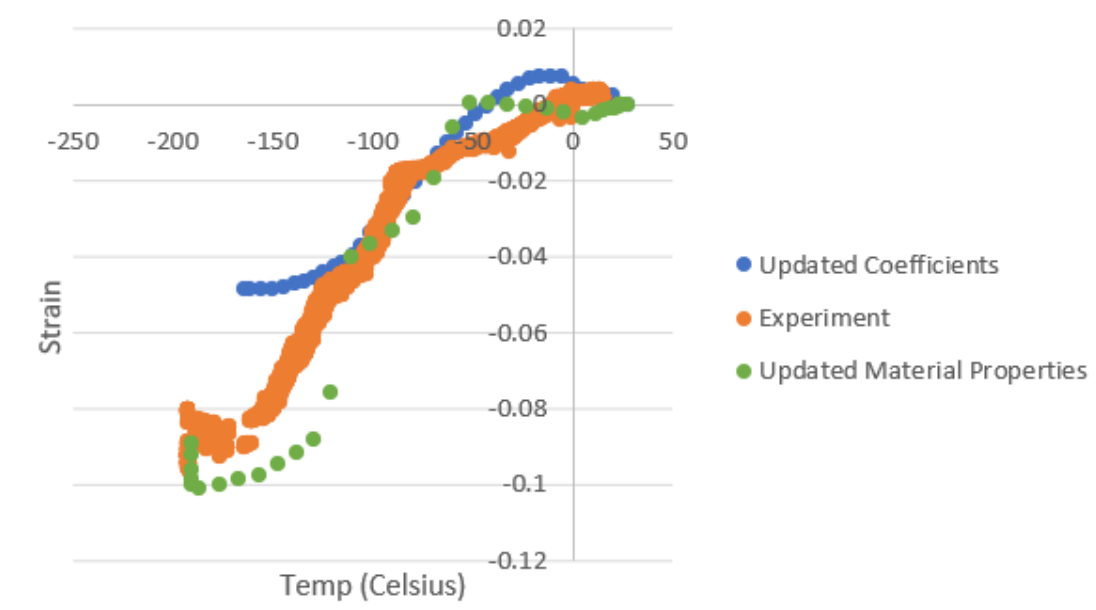

Figure 14: Comparison of cooldown strains in the diametral direction 


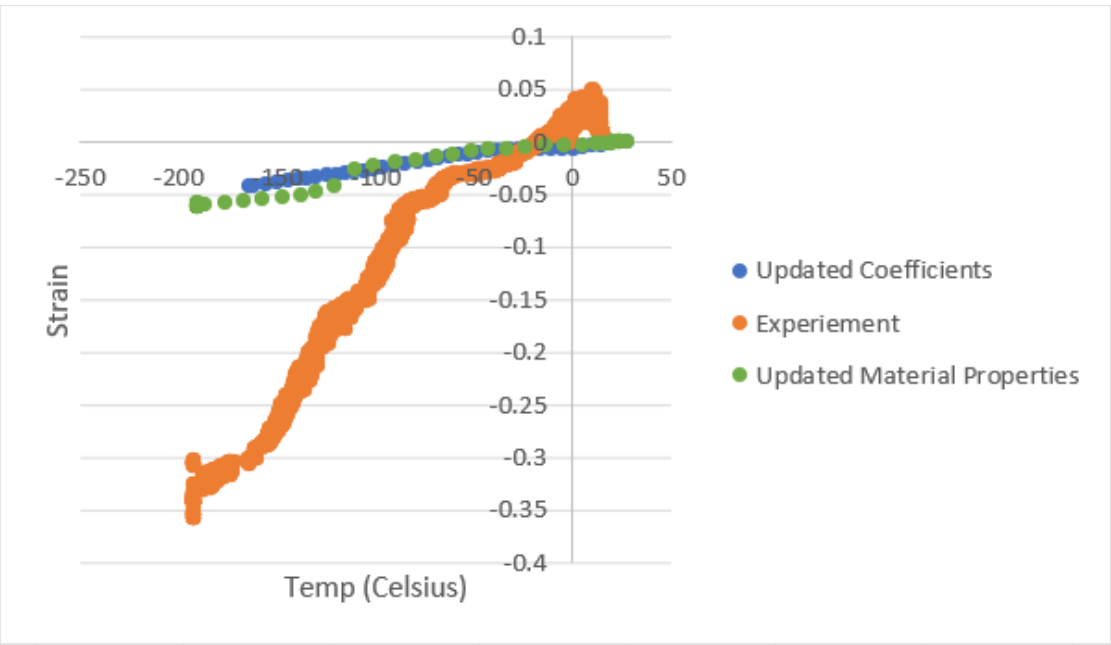

Figure 15: Comparison of cooldown strains in the circumferential direction

\section{Future Work/Conclusion}

The addition of a temperature thermal expansion coefficient helped the model in the Cooldown phase, where thermal strains are more dominant. Normalization of the Young's Modulus and Poison's Ratio helped the simulation nearly match the total experimental strain in the Pressurization phase. The effect of changing these material properties were not constrained only to the Pressurization phase though. The Cooldown phase showed significant improvement in the diametral direction, but the axial strain is overestimated. In order to further perfect the model, material properties need to be further refined and some parts of the experimental apparatus, like the rings that hold the tank in place, may need to be modeled. After the Cooldown phase and Pressurization are modeled satisfactorily, the next phases in the experiment will be modeled. Once the RTV-655 tank model is complete and confidence in the material properties is justified, the RTV/Aerogel tank will be modeled and compared to experimental results.

\section{References}

1] J. G. Marchetta, F. Sabri, D. S. Williams, and D. W. Pumroy, "Small-Scale RTV-655/Aerogel Cryogenic Liquid Storage Tank for Space Applications," Journal of Spacecraft and Rockets

2] J. G. Marchetta, and F. Sabri, "Development, Characterization, and Validation of an Aerogel/RTV Based Cryogenic Propellant Tank"

3] R. J. Corruccini, and John J. Gniewek, "Thermal Expansion of Technical Solids at Low Temperatures," National Bureau of Standards Monograph 29, pp. 15

4] N.A., "Thermal Expansion," Abaqus Analysis User's Guide"

5] J. Liska, "Effect of Low Temperatures on Young's Modulus of Elastomers", Industrial and Engineering Chemistry,

Vol. 36 No. 1

6] S. Pandini, and A. Pegoretti, "Time and Temperature Effects of Poisson's Ratio of poly(butylene terephthalate)", Express Polymer Letters, Vol. 5, No.8 